López-Rodríguez, M.M.; Aguilera-Vásconez, R.P.; Fernández-Sola, C.; Hernández-Padilla J.M.; Jiménez-Lasserrotte M.M. y Granero-Molina J. (2020) Sexual Desire and Excitement in Relation to Physical Activity in Young Adults. Revista Internacional de Medicina y Ciencias de la Actividad Física y el Deporte vol. 20 (77) pp. 133-153 Http://cdeporte.rediris.es/revista/revista77/artdeseo1135.htm DOI: $10.15366 /$ rimcafd2020.77.009

\title{
DESEO Y EXCITACIÓN SEXUAL EN RELACIÓN CON LA ACTIVIDAD FÍSICA EN JÓVENES ADULTOS
}

\section{SEXUAL DESIRE AND EXCITEMENT IN RELATION TO PHYSICAL ACTIVITY IN YOUNG ADULTS}

López-Rodríguez, M.M. ${ }^{1}$; Aguilera-Vásconez, R.P. ${ }^{2}$; Fernández-Sola, C. ${ }^{3}$; Hernández-Padilla J.M. ${ }^{3}$; Jiménez-Lasserrotte M.M. ${ }^{4}$ y Granero-Molina J. ${ }^{5}$

1 PhD. Grupo de Investigación de Ciencias de la Salud CTS-451. Universidad de Almería (España). mlr295@ual.es

2 Psicóloga Clínica. Máster en Ciencias de la Sexología. Universidad de Almería (España) natiaguileravas@gmail.com

3 PhD. Departamento Enfermería, Fisioterapia y Medicina. Universidad de Almería (España). cfernan@ual.es, j.hernandez-padilla@ual.es

${ }^{4}$ PhD. Cruz Roja Española. Almería (España). mariil@cruzroja.es

${ }^{5}$ PhD. Grupo de Investigación de Ciencias de la Salud CTS-451. Universidad de Almería (España). igranero@ual.es

Código UNESCO / UNESCO Code: 6199 Otras especialidades psicológicas (psicología del deporte) Other psycological specialities (Sport Psycology)

Clasificación del Consejo de Europa / Classification of the Council of Europe: 15 Psicología del Deporte / Sports Psychology

Recibido 25 de mayo de 2018 Received May 25, 2018

Aceptado 31 de agosto de 2018 Accepted August 31, 2018

\section{RESUMEN}

El deseo sexual es un estado de motivación que puede inducir a la actividad sexual. La actividad física está relacionada con el bienestar en social y sexual. El objetivo del presente estudio fue analizar el deseo sexual en relación con la actividad física y su impacto en la excitación e inhibición sexual en una población de jóvenes adultos. La muestra estuvo conformada por 485 participantes (327 mujeres y 158 hombres), entre 18 y 35 años. Este estudio requirió el cumplimiento de un cuestionario sociodemográfico, Inventario de Deseo Sexual (diádico y solitario), Cuestionario de Actividad Física (IPAQ) y Escalas de Inhibición y Excitación Sexual (SES-SIS). Los principales resultados mostraron relación entre el deseo y la 
actividad física con la excitación y la inhibición sexual, concluyendo que niveles de actividad medios y altos contribuyen de forma positiva en la sexualidad. Futuras investigaciones podrían proponer el ejercicio como intervención en problemas de deseo.

PALABRAS CLAVE: Deseo Sexual; Actividad física; Conducta Sexual; Salud Sexual

\section{ABSTRACT}

Sexual desire is a state of motivation that can induce to participate in sexual activity. Physical activity is related to the well-being in both social and sexual relationships. The aim of this study was to analyze sexual desire in relation to physical activity and its impact on sexual excitation and inhibition in a population of young adults. The total sample consisted of 485 participants (327 women and 158 men), aged between 18 and 35 years. This study required the fulfillment of a sociodemographic questionnaire, the Sexual Desire Inventory (dyadic and solitary), the Physical Activity Questionnaire (IPAQ) and the Sexual Inhibition and Sexual Excitation Scales (SES-SIS). The main results showed that there is a relationship between desire and physical activity with excitement and sexual inhibition, concluding that medium and high activity levels contribute positively in people's sexual behavior. Future research could propose exercise as an intervention in problems of desire.

KEYWORDS: Sexual Desire; Physical activity; Sexual Behavior; Sexual Health.

\section{INTRODUCCIÓN}

La sociedad constituye el eje central en la implantación de estereotipos de belleza y así, en las sociedades occidentales, los cuerpos delgados son un indicador de buena salud y de atractivo sexual. Debido a esto, algunas personas intentan aproximarse a estos cánones por medio de la realización de actividad física o intervenciones estéticas. (Penhollow y Young, 2008; Sacamori, Felizola, Kruguer, Sperandio y Cardoso, 2013; Uhlmann, Donovan, Zimmer-Gembeck, Bell, y Ramme, 2018). Esto conduce a considerar el efecto que estas prácticas ejercen sobre el sexo opuesto y cómo la distorsión de la imagen corporal afecta al deseo sexual (Haavio-Mannila y Purhonen, 2001).

El deseo sexual es un estado de motivación que puede inducir a las personas a buscar o participar en una actividad sexual (Corona et al., 2013). Este deseo, provocado por la interacción entre los procesos cognitivos internos, neurofisiológicos y componentes afectivos, que harán perpetuar la especie o provocar placer en el sujeto que la experimenta (Corona et al., 2013), se puede reconocer en dos formas: el deseo sexual solitario, que es un tipo de deseo que no involucra a un compañero e implica abstenerse de la intimidad con los 
demás, y el deseo sexual diádico, relacionado con la cercanía e intimidad con el otro (Ortega, Zubeidat y Sierra, 2006; Dosch, Ghisletta, y Van der Linden, 2016). A nivel hormonal, la comunicación endocrina es importante en el campo diádico de la medicina sexual ya que puede afectar al cerebro sexual (mediante el deseo) o al cuerpo sexual (a través de la excitación, erección/lubricación) (Corona, Isidori, Aversa, Burnett y Maggi, 2016).

La imagen corporal de mujeres y hombres, acompañada con variables de aptitud física ha demostrado ser predictor significativo de la satisfacción sexual (Penhollow y Young, 2008; Pujols, Meston y Seal, 2010; Sacamori et al., 2013). Estas percepciones o sentimientos sobre el cuerpo, tienen influencias socioculturales, grupales, e intrapersonales tan fuertes que pueden afectar a las experiencias sexuales y la salud de las personas (Mor, Pujols et al., 2010; Parfionov, Davidovitch y Grotto 2014). Así, la percepción de una imagen corporal deficiente relacionada con una menor eficiencia, asertividad y estima sexual, conduciría a la evitación sexual, y a un menor deseo diádico y solitario (Dosch et al., 2016). Por el contrario, una imagen corporal positiva asociada a una mayor frecuencia de actividad sexual, aventura, oportunismo y funcionamiento (Koyuncu, Tok, Canpolat y Catikkas, 2010; Pujols et al., 2010), conduciría a experiencias sexuales más frecuentes, sentirse deseables, y tener menos dificultades en este campo (Huang, Lee y Chang, 2007; Penhollow y Young, 2008).

Haciendo referencia a cómo impacta la percepción de uno mismo en el deseo sexual, se deben considerar la excitación e inhibición sexuales. Según el modelo de Control Dual de la respuesta sexual, la excitación sexual es el resultado total del balance entre la excitación y la inhibición sexual, siendo los dos, además, independientes de existir y necesarias para la adaptación (Bancroft, Graham, Janssen, y Sanders, 2009). En relación a esto, aquellos individuos con altos niveles de excitación se han relacionado con conductas sexuales de riesgo, e individuos con altos niveles de inhibición, con una mayor posibilidad de padecer una disfunción sexual (Granados, Salinas y Sierra, 2017; Moyano y Sierra, 2014).

Son varias las investigaciones que resaltan los beneficios encontrados a largo plazo en la realización del ejercicio físico, (Kulig, Brener y McManus, 2003; Jiménez, Zagalaz, Molero, Rulido y Ruiz, 2013) mediante la prevención de enfermedades o la mejora del funcionamiento cognitivo, articular y muscular (Adeniyi, Adeleye, y Adeniyi, 2010; Corona et al., 2016; La Vignera, Condorelli, Vicari, D’Agata y Calogero, 2011; La Vignera, Condorelli, Vicari, D’Agata y Calogero, 2012; Hackney, Lane, Register-Mihalik y O’Leary, 2017; Sgrò y Di Luigi, 2017; Stojanovska, Apostolopoulos, Polman y Borkoles, 2014).

A pesar de estos beneficios, la participación deportiva de tipo competitivo se ha relacionado con un inicio sexual tardío (Miller, Sabo, Farrell, Barnes y Melnick, 1998), y la de tipo lúdico, con un inicio precoz en las relaciones sexuales (Nogueira, Wijtzes, van de Bongardt, van de Looij-Jansen, Bannink y Raat, 2016) mientras que la práctica de alto rendimiento se ha llegado a relacionar con problemas de 
sexualidad (Sgrò y Di Luigi, 2017). Así, se ha señalado que las mujeres deportistas de alto rendimiento refieren insatisfacción de la imagen corporal y ansiedad de cuerpo social (Koyuncu et al., 2010) relacionando su participación en los deportes con una menor actividad sexual y riesgo de embarazo (Habel, Dittus, De Rosa, Chung y Kerndt, 2010; Miller et al., 1998; Sgrò y Di Luigi, 2017). Del mismo modo, en ambientes deportivos de tipo competitivo, los hombres también podrían generarse dudas sobre su físico, que podría influir negativamente en el comportamiento sexual (Sgrò y Di Luigi, 2017). A su vez, varios estudios (Habel et al., 2010; Miller et al., 1998; Taliaferro, Rienzo y Donovan, 2010) señalan una menor actividad sexual en las mujeres atletas, mientras que en los hombres ocurría lo contrario. Otros estudios (Lane, Register-Mihalik, O'Leary y Hackney, 2016; Sgrò y Di Luigi, 2017) consideran que altos niveles de entrenamiento contribuyen a la disminución de la libido en los hombres y a la formación de espermatozoides anormales, mientras que en las mujeres provocaría amenorreas, disfunciones menstruales o infertilidad (Hackney et al., 2017) suponiendo esto sobreadaptaciones fisiológicas a nivel físico que conllevan ahorro de recursos bioenergéticos (menor líbido).

Por el contrario, ciertas investigaciones coinciden en que las personas que practican actividad física de tipo competitivo pueden evitar el consumo de alcohol, drogas o actividades sexuales por mantenerse enfocados en su rendimiento (Kulig et al., 2003), lo que conllevaría a alejarse de situaciones de riesgo (Habel et al., 2010; Sgrò y Di Luigi, 2017). Además, se ha señalado que el ejercicio mejora la función eréctil en hombres sanos, y jóvenes (Hsiao et al., 2012; La Vignera et al., 2011; La Vignera et al., 2012; Sgrò y Di Luigi, 2017), impactando en su impulso sexual, función orgásmica y satisfacción (Sgrò y Di Luigi, 2017), mientras que el yoga en las mujeres potencia el deseo, excitación, lubricación, orgasmo, y satisfacción sexual (Finley, 2017). A su vez, la participación deportiva se ha relacionado también con una mayor competencia social, habilidades de resolución de problemas, autoestima, autoeficiencia, logros académicos (Taliaferro et al., 2010; Stojanovska et al., 2014) y bienestar general (Huang et al., 2007; Penhollow y Young, 2008), así como mayores niveles de felicidad y satisfacción con la vida (Jiménez et al., 2013; Sacomori, Felizola, Kruguer, Sperandio, y Cardoso, 2013).

A nivel médico, se ha señalado que la actividad física mejora la función sexual de mujeres deprimidas (Lorenz y Meston, 2012; Lorenz y Meston, 2013; Finley, 2017; Stojanovska et al., 2014), mejorando síntomas de tristeza (Sgrò y Di Luigi, 2017) y ansiedad (Huang et al., 2007). Del mismo modo, el ejercicio ha mostrado ser efectivo como tratamiento para la disfunción eréctil (Esposito et al., 2009; La Vignera et al., 2012; Sgrò y Di Luigi, 2017) o para mejorar niveles de disfunción sexual, peso, y testosterona (Khoo et al., 2013), inclusive como acompañamiento de inhibidores de la fosfodiesterasa-5 (iPDE 5) que suelen constituir el tratamiento de la disfunción eréctil (Maio, Saraeb y Marchiori, 2010). Por el contrario, el sedentarismo ha mostrado una influencia negativa en la salud en general, y en la salud sexual en particular (La Vignera et al., 2011; Sgrò y Di Luigi, 2017). 
En cuanto a los instrumentos utilizados para la valoración de la actividad física, algunos estudios optan por utilizar ítems específicos no validados (Miller et al., 1998; Habel et al., 2010; Huang et al., 2007; Nogueira et al., 2016; Taliaferro et al., 2010) o diarios (Esposito et al., 2009; Skoyen, Blank, Corkery y Butler, 2013). Sin embargo, el cuestionario validado más utilizado en la literatura consultada para esta investigación, fue el Cuestionario Internacional de Actividad Física (IPAQ) (CrespoSalgado, Delgado-Martín, Blanco-Iglesias y Aldecoa-Landesa, 2015; Sacomori et al., 2013; Sigmundová et al., 2015, Hackney et al., 2017).

Por tanto, y debido al vínculo demostrado entre actividad física y sexualidad (Sgrò y Di Luigi, 2017), este estudio se propone analizar el deseo sexual en relación con la actividad física y su impacto en la inhibición o excitación sexual en una población de jóvenes adultos. De este modo se pretende conocer el efecto del ejercicio físico sobre el deseo sexual y la inhibición/desinhibición sexual. Además, como objetivo específico, se analizan en este estudio posibles diferencias en estas variables en relación al género y la edad de los participantes.

Por lo tanto, nos planteamos como hipótesis la existencia de puntuaciones significativamente mayores en deseo y excitación sexual en individuos con un nivel de ejercicio medio o alto, con respecto a aquellos con un nivel de actividad bajo.

\section{MÉTODO}

\section{Participantes}

En el presente estudio correlacional participaron 485 personas (327 mujeres y 158 hombres) de edades comprendidas entre 18 a 35 años, con una media de $22,84 \pm 3,22$ en hombres y $22,25 \pm 3,44$ en las mujeres. El $36,5 \%(n=177)$ de los sujetos tenían 20 años o menos, mientras que otro $35,9 \%(n=174)$ se situaba entre los 21 y 23 años. El 16,3\% ( $n=79)$ tenían entre 24 y 26 años, el 7,2\% $(n=35)$ entre los 27 y 29 años, y el $4,1 \%(n=20)$ restante contaba con 30 años o más. Para el reclutamiento de los participantes se utilizó un muestreo de conveniencia y se creó un cuestionario online, a través de la plataforma Google Drive, anunciándolo en redes sociales y webs universitarias (www.ual.es). El formulario estructurado contenía datos sociodemográficos, escalas de actividad física, deseo sexual, así como un inventario de excitación e inhibición sexual.

\section{Componentes de los instrumentos}

Sociodemográfico.

El cuestionario sociodemográfico recogió datos como edad, sexo, nivel de estudios y existencia de una pareja estable (al menos 3 meses de relación) (Anexo). 
Rev.int.med.cienc.act.fís.deporte - vol. 20 - número 77 - ISSN: 1577-0354

\section{Actividad Física}

Se aplicó el Cuestionario Internacional de Actividad Física (IPAQ) (www.ipaq.ki.se) en su versión corta, consistente en 7 preguntas que valoran la actividad física realizada. Este instrumento fue elegido debido a que permite estimar el tiempo semanal invertido en la realización de actividades físicas de forma sencilla, habiendo sido anteriormente utilizado en este tipo de estudios (Sacomori et al., 2013; Sigmundová et al., 2015; Hackney et al., 2017) así como en el Eurobarómetro de la Comisión Europea (European Opinion Research Group, 2003). Así mismo, se utilizó la versión corta de este cuestionario con el objetivo de facilitar su realización a los participantes. Dicha versión se encuentra además validada en población española (Román-Viñas, Ribas-Barba, Ngo, y Serra-Majem, 2013) con una correlación moderada para actividad física total $(r=0,27 ; p<.05)$ y tiempo dedicado a actividad física vigorosa $(r=0,38 ; p<.01)$, así como una sensibilidad y especificidad del $75 \%(K=0,33)$.Las preguntas de este cuestionario están relacionadas con tareas deportivas, laborales y de sedentarismo, distinguiendo así entre tres factores como son caminata, actividades físicas moderadas y actividades físicas vigorosas (Crespo-Salgado et al., 2015). Para determinar los niveles de IPAQ se multiplicaron los minutos de ejercicio por el total de días en que se realiza, obteniendo así la puntuación total. Bajo esta puntuación se clasificó a los participantes en: Nivel de Actividad Bajo $(<600)$, Nivel de Actividad Medio (entre 600 y 3000) y Nivel de Actividad Alto ( $>3000)$. En nuestra muestra se obtuvo un alfa de Cronbach de 0,80 y una correlación ítem-total corregida entre 0.72 y 0.80 . Por su parte, el análisis de fiabilidad mostró coeficientes de correlación de Spearman entre 0,33 y 0,72.

\section{Deseo Sexual}

El deseo sexual de los participantes se evaluó a través del Inventario de Deseo Sexual de Spector, Carey y Steinberg, (1996), adaptado al español por Ortega, Zubeidat y Sierra (2006). Este inventario mide el deseo sexual de las personas a través de 13 preguntas que evalúan el nivel de deseo sexual de la persona. Este instrumento distingue dos factores, deseo sexual diádico (ítem 1-9) que se refiere a actividades sexuales en las que se involucra a una pareja, y deseo sexual solitario (ítem 10-13) que se refiere al interés en actividades sexuales que no involucran a una pareja. Todos los ítems puntúan mediante una escala tipo Likert. La adaptación española presenta valores alfa de Cronbach óptimos, así como una validez adecuada ( 0.87 para el deseo sexual diádico y 0.88 para el deseo sexual solitario) y una correlación entre los dos factores de 0,49 (Ortega et al., 2006). Además, se determinó el nivel de Deseo Sexual utilizando 45 como punto de corte ("Sexual Desire Quiz. Maze Women's Sexual Health", 2018), y clasificando la muestra por encima de dicho punto de corte con un Nivel de Deseo Sexual Normal, y por debajo con un Nivel de Deseo Sexual Bajo (Spector et al., 1996). En el presente estudio se obtuvo un alfa de Cronbach de 0,85 y una correlación ítem-total corregida entre 0.53 y 0.90 . Por otra parte, los coeficientes de correlación de Spearman entre los ítems se sitúan entre 0,34 y 0,83. 


\section{Excitación / Inhibición sexual}

Se aplicó la versión española, de las Escalas de Inhibición Sexual/Excitación Sexual- Forma Breve (SIS/SES-SF; Carpeter, Janssen, Graham, Vorts y Wichertas, 2011), adaptada y validada por Moyano y Sierra (2014). Este inventario consta de 14 preguntas calificadas en escala Likert de 4 puntos, que miden tres subescalas: La Escala de Excitación Sexual (SES) que evalúa por interacciones sociales, la Escala de Inhibición Sexual 1 (SIS1) con ítems relacionados con la distracción o concentración en el acto sexual, y la Escala de Inhibición Sexual 2 (SIS2) que evalúa el miedo del sujeto a ser descubierto o a contagiarse por una infección de transmisión sexual (Moyano y Sierra, 2014). De acuerdo a la puntuación obtenida, se clasificó a los participantes en los diferentes niveles de excitabilidad (SES, SIS1 y SIS2). Aquellos participantes con igual puntuación en dos escalas, se agruparon en: SES-SIS1; SES-SIS2 y SIS. Los indicadores de fiabilidad de la versión en castellano fueron adecuados en ambos sexos, alcanzándose valores alfa de Cronbach que oscilan entre 0,60 de SIS2 y 0,72 de SES, y las correlaciones ítem-total corregidas en cada factor oscilaron entre 0,28 y 0,64. Así mismo, se obtuvieron correlaciones significativas entre los factores, excepto entre SES y SIS1, demostrándose independencia entre ellos (Moyano y Sierra, 2014) En el presente estudio se obtuvo un alfa de Cronbach entre 0,69 de SIS1 y 0,75 de SES, y una correlación ítem-total corregida de cada factor entre 0,30 y 0,77 . Por otra parte, los coeficientes de correlación entre SES y SIS1 muestran también en nuestro estudio que dichos factores son independientes.

\section{PROCEDIMIENTO}

La evaluación se realizó a través de un cuestionario online, creado en la plataforma Google Drive, cuyo enlace fue distribuido en diversas redes sociales. La muestra fue recogida entre los meses de diciembre 2017 y marzo 2018. Cuando los participantes accedían al enlace se les presentó el consentimiento informado del estudio con las instrucciones para realizarlo. La participación fue voluntaria, sin ningún tipo de compensación. Además, tres cuestiones actuaban como filtro muestral, permitiendo o no que el participante pudiera continuar realizando el cuestionario online. En primer lugar la persona debía tener entre 18 y 35 años de edad. Además debía contestar de forma negativa a dos cuestiones relativas a la existencia de limitación física y de enfermedad crónica que impidieran o afectaran de algún modo a la realización de actividad física.

Para la realización del estudio se obtuvo la aprobación del comité de Ética de la Universidad de Almería. Al inicio del cuestionario se informó a los individuos sobre los propósitos de la investigación, así como el carácter voluntario y anónimo de su participación. Todos los participantes dieron su consentimiento para la realización del estudio. El anonimato y la confidencialidad se aseguraron durante la recopilación y el análisis de datos, bajo los tratados de legislación europea sobre protección de datos (COM, 2012) 


\section{ANÁLISIS ESTADÍSTICO}

Todos los análisis estadísticos se llevaron a cabo con el programa SPSS v. 23.0 para Windows (SPSS Inc., Chicago, IL, USA). Los datos cuantitativos se muestran como media y desviación estándar, y los datos cualitativos como frecuencias y porcentajes. Se verificó que todas las variables se ajustaban a la normalidad mediante la prueba KS. Las variables continuas se analizaron mediante pruebas $t$ de Student y ANOVA, utilizando test de Bonferroni para el análisis post hoc, y eta cuadrado para el análisis del tamaño del efecto. Para el estudio de las variables categóricas se utilizó el test de chi-cuadrado. Las correlaciones se analizaron mediante el método de Pearson.

\section{RESULTADOS}

Para empezar se realizó un análisis univariante de los datos obtenidos, encontrando una media de edad del total de la muestra de 22,44 $\pm 3,37$ años. Así mismo, el 50,5\% de la población refirió tener pareja durante al menos tres meses en el momento del estudio, mientras que el $40,0 \%$ afirmaba no tenerla y el 9,5\% manifestaba algún tipo de relación íntima durante ese intervalo de tiempo, pero sin ser considerado pareja. Según la puntuación total en la escala de deseo sexual se clasificó a un $84,7 \%$ de la población en un nivel de deseo sexual normal, frente a un 15,3\% clasificado con un nivel de deseo sexual bajo. Los niveles de IPAQ determinaron una mayoría de participantes con nivel de actividad alto $(45,6 \%)$, seguido de un nivel de actividad medio (34,2\%). En las escalas de excitabilidad sexual, un $60,2 \%$ de la población mostró la mayor puntuación en SES y un $27,4 \%$ en SIS2.

Al comparar las variables entre hombres y mujeres se observaron diferencias significativas en las puntuaciones de actividad moderada, actividad vigorosa $(p<.05)$, con medias superiores al grupo de hombres. En relación a este dato se apreciaron también diferencias significativas entre sexos para el nivel de actividad $(\mathrm{p}<.05)$, encontrando que el $22,9 \%$ de las mujeres poseían un nivel de actividad bajo, frente al $14,6 \%$ de los hombres. Por otro lado, los hombres mostraron también puntuaciones significativamente mayores en las escalas de deseo y SES $(p<.05)$. Sin embargo, la puntuación en SIS2 obtuvo medias significativamente más altas $(p<.05)$ entre las mujeres. Del mismo modo, se observó también un mayor porcentaje de mujeres con pareja (53,80\%) (Tabla 1$)$. 
Rev.int.med.cienc.act.fís.deporte - vol. 20 - número 77 - ISSN: 1577-0354

Tabla 1. Comparación de las variables según el sexo de los participantes.

\begin{tabular}{|c|c|c|c|c|c|c|c|c|c|}
\hline & & \multicolumn{2}{|c|}{$\begin{array}{c}\text { Hombres } \\
n=158\end{array}$} & \multicolumn{2}{|c|}{$\begin{array}{c}\text { Mujeres } \\
\mathrm{n}=327\end{array}$} & \multicolumn{2}{|c|}{$\begin{array}{l}\text { Prueba } \\
\text { Levene }\end{array}$} & \multirow[b]{2}{*}{$\mathrm{t}$} & \multirow[b]{2}{*}{$p$} \\
\hline & & $\mathrm{M}$ & D.E & $\mathrm{M}$ & D.E & $\mathrm{F}$ & $p$ & & \\
\hline \multicolumn{2}{|l|}{ Edad } & 22,84 & 3,22 & 22,25 & 3,44 & 0,68 & .40 & 1,81 & .07 \\
\hline \multicolumn{2}{|l|}{ Caminata } & $2.049,91$ & $3.114,24$ & $1.537,02$ & $2.225,27$ & 8,28 & .01 & 1,85 & .06 \\
\hline \multicolumn{2}{|c|}{ Actividad Moderada } & $1.455,24$ & $3.437,54$ & 742,67 & $1.787,31$ & 14,13 & .00 & 2,45 & .01 \\
\hline \multicolumn{2}{|c|}{ Actividad Vigorosa } & $2.304,05$ & $3.867,26$ & 1538,32 & $3.922,99$ & 1,45 & .22 & 2,02 & .04 \\
\hline \multicolumn{2}{|l|}{ IPAQ } & $5.809,20$ & $7.657,46$ & 3818,01 & $6.256,96$ & 6,82 & .01 & 2,84 & .00 \\
\hline \multicolumn{2}{|l|}{ Deseo } & 66,65 & 14,70 & 55,16 & 16,66 & 2,51 & .11 & 7,39 & .00 \\
\hline \multicolumn{2}{|c|}{ Deseo Diádico } & 49,03 & 10,72 & 43,67 & 11,80 & 0,12 & .72 & 4,82 & .00 \\
\hline \multicolumn{2}{|c|}{ Deseo Solitario } & 17,65 & 6,75 & 11,47 & 8,58 & 21,08 & .00 & 8,61 & .00 \\
\hline \multicolumn{2}{|l|}{ SES } & 14,32 & 3,27 & 12,83 & 3,60 & 1,97 & .16 & 4,37 & .00 \\
\hline \multicolumn{2}{|l|}{ SIS1 } & 8,02 & 2,46 & 8,37 & 2,55 & 0,22 & .63 & $-1,43$ & .15 \\
\hline \multirow{2}{*}{\multicolumn{2}{|c|}{ SIS2 }} & 11,19 & 2,82 & 11,88 & 3,09 & 1,44 & .23 & $-2,34$ & .02 \\
\hline & & Frec. & $\%$ & Frec. & $\%$ & & & $X^{2}$ & $p$ \\
\hline \multirow[t]{3}{*}{ Nivel IPAQ } & Bajo & 23 & $14,6 \%$ & 75 & $22,9 \%$ & & & 9,35 & .01 \\
\hline & Medio & 48 & $30,4 \%$ & 118 & $36,1 \%$ & & & & \\
\hline & Alto & 87 & $55,1 \%$ & 134 & $41 \%$ & & & & \\
\hline \multirow[t]{2}{*}{ Nivel Deseo } & Bajo & 10 & $6,3 \%$ & 64 & $19,6 \%$ & & & 14,45 & .00 \\
\hline & Normal & 148 & $93,7 \%$ & 263 & $80,4 \%$ & & & & \\
\hline \multirow[t]{6}{*}{ Nivel Exc. } & SES & 118 & $74,7 \%$ & 174 & $53,2 \%$ & & & 21,26 & .00 \\
\hline & SES-SIS1 & 0 & $0,0 \%$ & 2 & $0,6 \%$ & & & & \\
\hline & SES-SIS2 & 9 & $5,7 \%$ & 30 & $9,2 \%$ & & & & \\
\hline & SIS & 1 & $0,6 \%$ & 4 & $1,2 \%$ & & & & \\
\hline & SIS1 & 2 & $1,3 \%$ & 12 & $3,7 \%$ & & & & \\
\hline & SIS2 & 28 & $17,7 \%$ & 105 & $32,1 \%$ & & & & \\
\hline \multirow[t]{3}{*}{ Pareja } & Con Pareja & 69 & $43,7 \%$ & 176 & $53,8 \%$ & & & 6,29 & .04 \\
\hline & Sin Pareja & 68 & $43 \%$ & 126 & $38,5 \%$ & & & & \\
\hline & $\begin{array}{l}\text { Otras } \\
\text { relaciones }\end{array}$ & 21 & $13,3 \%$ & 25 & $7,6 \%$ & & & & \\
\hline
\end{tabular}


Así mismo se analizaron posibles diferencias entre la población con y sin pareja, obteniendo diferencias sólo para el deseo solitario $(p<.01)$ con medias superiores para el grupo sin pareja.

A continuación se realizó un análisis de diferencia de medias en función de subniveles de edad. Para ello, y para que el número de integrantes de los grupos fuera comparable, se consideró un grupo que reunía a la muestra mayor de 27 años. En este análisis la variable relativa a tener pareja mostró diferencias significativas, encontrando un mayor porcentaje de sujetos con pareja entre el grupo mayor de 27 años. Las variables SES y deseo junto con sus dos subescalas obtuvieron también diferencias significativas. Así, el análisis post hoc de SES señaló diferencias entre el grupo de 21 a 23 años con respecto al grupo de más de 27 años $(p<.01)$. Por otra parte, las diferencias significativas en el deseo y sus subescalas, se encontraron entre los dos grupos de menor edad, con respecto al grupo de más de 27 años ( $p<.01$ en ambos casos) (Tabla 2). 
Rev.int.med.cienc.act.fís.deporte - vol. 20 - número 77 - ISSN: 1577-0354

Tabla 2. Comparación de las variables según grupo de edad de los participantes.

\begin{tabular}{|c|c|c|c|c|c|c|c|c|c|c|c|c|c|}
\hline & & \multicolumn{2}{|c|}{$\begin{array}{c}18-20 \text { años } \\
n=177\end{array}$} & \multicolumn{2}{|c|}{$21-23$ años $n=174$} & \multicolumn{2}{|c|}{24 y 26 años $n=79$} & \multicolumn{2}{|c|}{$\begin{array}{c}27-35 \text { años } \\
n=55\end{array}$} & \multicolumn{2}{|c|}{$\begin{array}{l}\text { Prueba } \\
\text { Levene }\end{array}$} & \multirow[b]{2}{*}{$F$} & \multirow[b]{2}{*}{$p$} \\
\hline & & $\mathrm{M}$ & D.E & $M$ & D.E & $M$ & D.E & $\mathrm{M}$ & D.E & $F$ & $p$ & & \\
\hline \multicolumn{2}{|l|}{ Caminata } & 1710,87 & 2605,66 & 1486,74 & 1797,76 & $F$ & $p$ & 1680,00 & 2928,86 & 3,17 & .02 & 1,35 & .25 \\
\hline \multicolumn{2}{|c|}{ Actividad Moderada } & 1040,95 & 3314,00 & 714,83 & 1249,34 & 1540,66 & 2652,61 & 771,64 & 1714,94 & 3,04 & .02 & 2,20 & .08 \\
\hline \multicolumn{2}{|c|}{ Actividad Vigorosa } & 1481,81 & 3532,60 & 1919,26 & 3923,33 & 2437,47 & 5337,81 & 1423,27 & 2300,22 & 1,74 & .15 & 1,31 & .27 \\
\hline \multicolumn{2}{|l|}{ IPAQ } & 4233,63 & 7598,53 & 4120,84 & 5375,04 & 6162,60 & 8497,26 & 3874,91 & 4948,47 & 2,36 & .07 & 2,00 & .11 \\
\hline \multicolumn{2}{|l|}{ Deseo } & 56,79 & 15,96 & 57,29 & 17,62 & 61,78 & 15,07 & 66,65 & 17,71 & 1,32 & .26 & 6,26 & .00 \\
\hline \multicolumn{2}{|c|}{ Deseo Diádico } & 43,60 & 11,28 & 45,30 & 12,43 & 46,86 & 10,68 & 49,55 & 11,19 & 0,86 & .46 & 4,18 & .01 \\
\hline \multicolumn{2}{|c|}{ Deseo Solitario } & 13,19 & 8,13 & 11,99 & 8,63 & 14,92 & 7,74 & 17,11 & 9,32 & 2,25 & .08 & 6,10 & .00 \\
\hline \multicolumn{2}{|l|}{ SES } & 13,36 & 3,28 & 12,68 & 3,74 & 13,73 & 3,36 & 14,60 & 3,75 & 2,05 & .10 & 4,68 & .00 \\
\hline \multicolumn{2}{|l|}{ SIS1 } & 8,48 & 2,41 & 8,07 & 2,43 & 7,94 & 2,59 & 8,64 & 3,00 & 1,44 & .23 & 1,62 & .18 \\
\hline \multirow{2}{*}{\multicolumn{2}{|c|}{ SIS2 }} & 11,94 & 2,87 & 11,74 & 3,03 & 10,94 & 3,01 & 11,51 & 3,34 & 1,07 & .36 & 2,11 & .10 \\
\hline & & Frec. & $\%$ & Frec. & $\%$ & Frec. & $\%$ & Frec. & $\%$ & & & $X^{2}$ & $p$ \\
\hline \multirow[t]{3}{*}{ Nivel IPAQ } & Bajo & 40 & $22,6 \%$ & 39 & $22,4 \%$ & 10 & $12,7 \%$ & 9 & $16,4 \%$ & & & 7,58 & .27 \\
\hline & Medio & 60 & $33,9 \%$ & 57 & $32,8 \%$ & 25 & $31,6 \%$ & 24 & $43,6 \%$ & & & & \\
\hline & Alto & 77 & $43,5 \%$ & 78 & $44,8 \%$ & 44 & $55,7 \%$ & 22 & $40,0 \%$ & & & & \\
\hline \multirow{2}{*}{$\begin{array}{l}\text { Nivel } \\
\text { Deseo }\end{array}$} & Bajo & 29 & $16,4 \%$ & 32 & $18,4 \%$ & 8 & $10,1 \%$ & 5 & $9,1 \%$ & & & 4,72 & .19 \\
\hline & Normal & 148 & $83,6 \%$ & 142 & $81,6 \%$ & 71 & $89,9 \%$ & 50 & $90,9 \%$ & & & & \\
\hline \multirow[t]{6}{*}{ Nivel Exc. } & SES & 108 & $61,0 \%$ & 92 & $52,9 \%$ & 54 & $68,4 \%$ & 38 & $69,1 \%$ & & & 22,99 & .08 \\
\hline & SES-SIS1 & 1 & $0,6 \%$ & 0 & $0,0 \%$ & 1 & $1,3 \%$ & 0 & $0,0 \%$ & & & & \\
\hline & SES-SIS2 & 18 & $10,2 \%$ & 18 & $10,3 \%$ & 2 & $2,5 \%$ & 1 & $1,8 \%$ & & & & \\
\hline & SIS & 1 & $0,6 \%$ & 4 & $2,3 \%$ & 0 & $0,0 \%$ & 0 & $0,0 \%$ & & & & \\
\hline & SIS1 & 5 & $2,8 \%$ & 3 & $1,7 \%$ & 4 & $5,1 \%$ & 2 & $3,6 \%$ & & & & \\
\hline & SIS2 & 44 & $24,9 \%$ & 57 & $32,8 \%$ & 18 & $22,8 \%$ & 14 & $25,5 \%$ & & & & \\
\hline \multirow[t]{3}{*}{ Pareja } & Con Pareja & 66 & $37,3 \%$ & 98 & $56,3 \%$ & 47 & $59,5 \%$ & 34 & $61,8 \%$ & & & 32,74 & .00 \\
\hline & Sin Pareja & 100 & $56,5 \%$ & 57 & $32,8 \%$ & 22 & $27,8 \%$ & 15 & $27,3 \%$ & & & & \\
\hline & $\begin{array}{l}\text { Otras } \\
\text { relaciones }\end{array}$ & 11 & $6,2 \%$ & 19 & $10,9 \%$ & 10 & $12,7 \%$ & 6 & $10,9 \%$ & & & & \\
\hline
\end{tabular}

Nota: $\mathrm{M}=$ Media; $\mathrm{D} . \mathrm{E}$ = Desviación estándar; Exc.=Excitabilidad; $p$ para la comparación de sexos $(F=$ Anova para variables continuas y $X^{2}=$ chi-cuadrado para variables categóricas). Nivel de Excitabilidad: SES-SIS1; SES-SIS2; SIS: Igual puntuación en ambas escalas.

Posteriormente se procedió a comparar los tres niveles de actividad según la puntuación en IPAQ (nivel de actividad alto, medio y bajo) encontrando diferencias significativas en deseo sexual, puntuación en SES y puntuación en SIS1. No obstante, los valores de eta cuadrado mostraron un tamaño del efecto débil para estas variables. Posteriormente, mediante análisis post hoc se observó que el grupo con nivel de actividad bajo obtuvo medias significativamente más bajas en la puntuación de deseo con respecto a los otros dos grupos $(p<.05)$. Del mismo modo, las medias en la puntuación SES del grupo con nivel de ejercicio bajo resultaron significativamente más bajas con respecto al grupo de actividad moderada $(p<.01)$ y al grupo de actividad alta $(p<.05)$. Sin embargo, la puntuación en SIS1 obtuvo una media más alta en el grupo con un nivel de actividad bajo, encontrando diferencias significativas con respecto al grupo con actividad alta $(p<.05)$. Además, se observaron diferencias significativas $(p<.05)$ en el porcentaje de sujetos con pareja, 
encontrando en el grupo con nivel de actividad bajo un 60,2\% de población con pareja. Por último, en cuanto al nivel de excitabilidad, en el grupo con nivel de actividad medio el $64,5 \%$ de los participantes tiene un SES más alto, frente a un $45,9 \%$ en el grupo de actividad baja (Tabla 3 ).

Tabla 3. Comparación por grupos según el nivel de IPAQ.

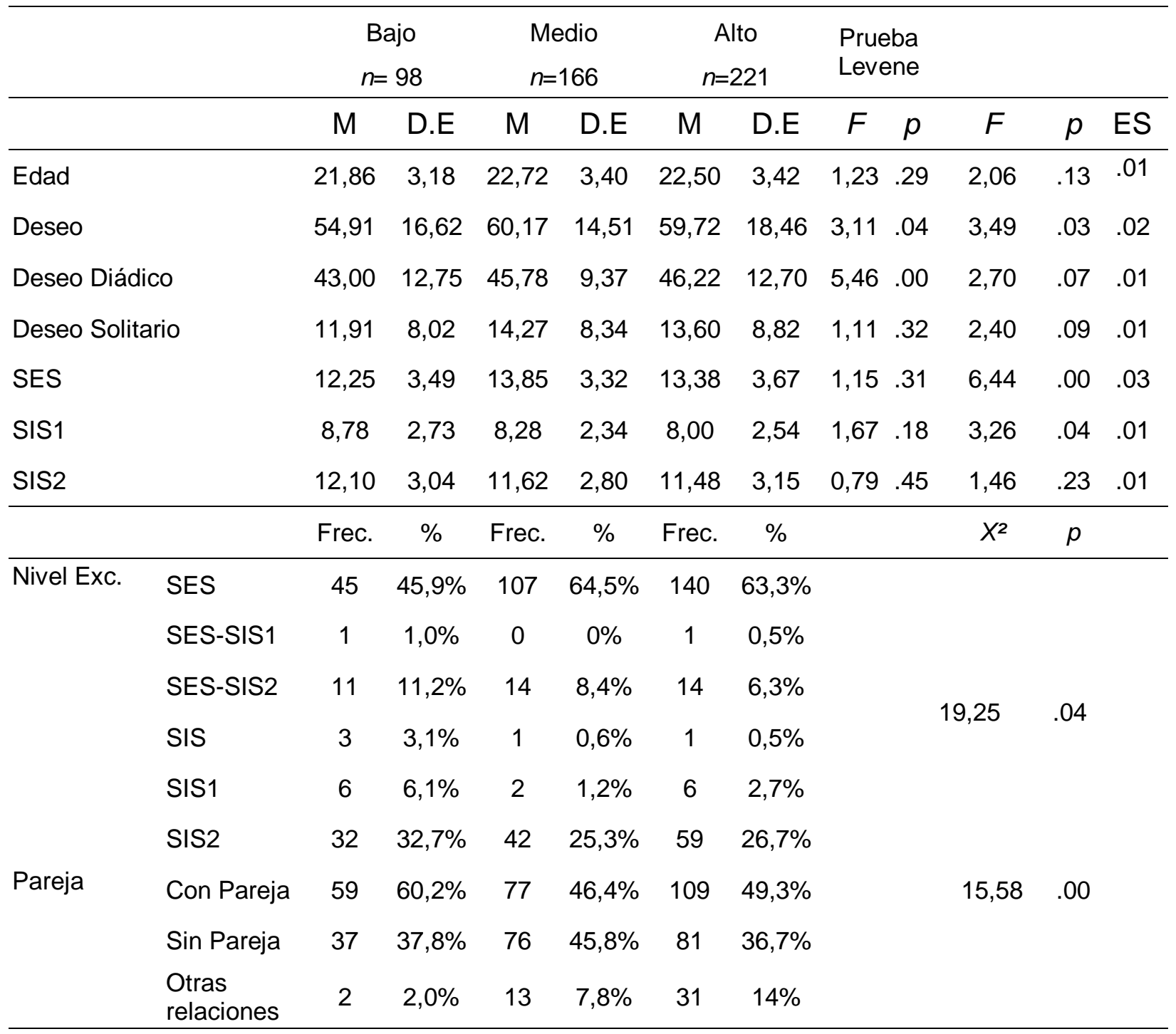

Nota. Exc.=Excitabilidad; ES=Tamaño del Efecto; $p$ para la comparación de variables $(F=$ Anova para variables continuas y $X^{2}=$ chi-cuadrado para variables categóricas). Nivel de Excitabilidad: SES-SIS1; SES-SIS2; SIS: Igual puntuación en ambas escalas.

Al analizar las correlaciones, se observó relación entre deseo (diádico y solitario) y variables como la edad $(p<.01)$, y la puntuación SES ( $p<.01)$. Además, se observó una correlación negativa entre el deseo solitario y la puntuación en SIS2 $(p<.01)$. Por otro lado, aunque la puntuación IPAQ no muestra correlación con las puntuaciones en la escala de deseo, se encontró que tanto dicha puntuación IPAQ 
como la actividad vigorosa se correlacionaron negativamente con la puntuación de SIS2 $(p<.05)($ Tabla 4).

Tabla 4. Correlación de las variables.

\begin{tabular}{|c|c|c|c|c|c|c|c|c|c|c|c|}
\hline & & Caminata & $\begin{array}{l}\text { Actividad } \\
\text { Moderada }\end{array}$ & $\begin{array}{l}\text { Actividad } \\
\text { Vigorosa }\end{array}$ & IPAQ & Deseo & $\begin{array}{l}\text { Deseo } \\
\text { Diádico }\end{array}$ & $\begin{array}{l}\text { Deseo } \\
\text { Solitario }\end{array}$ & SES & SIS1 & SIS2 \\
\hline Edad & Pearson & 0,01 & 0,02 & 0,02 & 0,03 & $0,16^{\star *}$ & $0,14^{\star \star}$ & $0,12^{*}$ & $0,11^{*}$ & 0,04 & $-0,06$ \\
\hline Caminata & Pearson & 1 & $0,43^{* *}$ & $0,24^{* *}$ & $0,67^{\star \star}$ & 0,00 & 0,01 & $-0,01$ & 0,06 & 0,02 & $-0,06$ \\
\hline $\begin{array}{l}\text { Actividad } \\
\text { Moderada }\end{array}$ & Pearson & & 1 & $0,41^{* *}$ & $0,77^{\star *}$ & 0,01 & 0,01 & 0,01 & 0,01 & $-0,07$ & $-0,07$ \\
\hline $\begin{array}{l}\text { Actividad } \\
\text { Vigorosa }\end{array}$ & Pearson & & & 1 & $0,82^{\star *}$ & $-0,04$ & $-0,02$ & $-0,06$ & $-0,08$ & $-0,05$ & $-0,11^{\star}$ \\
\hline IPAQ & Pearson & & & & 1 & $-0,02$ & $-0,00$ & $-0,03$ & $-0,02$ & $-0,05$ & $-0,11^{\star}$ \\
\hline Deseo & Pearson & & & & & 1 & $0,88^{* *}$ & $0,76^{\star *}$ & $0,57^{\star *}$ & $-0,02$ & $-0,08$ \\
\hline $\begin{array}{l}\text { Deseo } \\
\text { Diádico }\end{array}$ & Pearson & & & & & & 1 & $0,38^{* *}$ & $0,53^{* *}$ & $-0,04$ & $-0,02$ \\
\hline $\begin{array}{l}\text { Deseo } \\
\text { Solitario }\end{array}$ & Pearson & & & & & & & 1 & $0,40^{\star *}$ & 0,02 & $-0,12^{*}$ \\
\hline SES & Pearson & & & & & & & & 1 & 0,08 & $-0,08$ \\
\hline SIS1 & Pearson & & & & & & & & & 1 & $0,31^{* *}$ \\
\hline
\end{tabular}

Nota: Pearson para correlación de variables; ${ }^{\star} p<.05 ;{ }^{*} p<.01$

\section{DISCUSIÓN}

Los resultados del presente estudio ponen de manifiesto importantes hallazgos con respecto a la relación entre el ejercicio y la sexualidad en jóvenes adultos. Así, los datos obtenidos indican una relación entre el nivel de actividad física y el deseo, la excitación y la inhibición sexual.

Para empezar, aunque incluyó un número considerable de hombres, la muestra estuvo conformada principalmente por mujeres. El nivel de ejercicio en nuestra muestra de adultos jóvenes, con una mayoría de los sujetos con nivel medio y alto de actividad, refleja lo expuesto en el estudio Sigmundová et al. (2015), donde se señalaba un aumento de actividad física por parte de los adultos, obteniendo un $21,3 \%$, en el nivel de actividad física bajo, similar al $20,2 \%$ obtenido en el presente estudio.

Al comparar hombres y mujeres se encontraron diferencias significativas en relación con el ejercicio, coincidiendo con el estudio realizado por Jiménez-Torres, GodoyIzquierdo y Godoy (2012) donde, al igual que en nuestra muestra, la población de hombres realizaba más ejercicio físico (moderado y vigoroso) que las mujeres. 
Estos autores (Jiménez-Torres et al, 2012) relacionan este hecho en la población masculina con una mayor motivación intrínseca, e implicación en la actividad física.

De forma paralela al mayor porcentaje de mujeres con nivel de actividad bajo, encontramos que existió en este estudio un mayor porcentaje de mujeres con pareja. Esto puede estar relacionado con la investigación realizada por Davis, Sandberg, Bradford y Larson (2016), que explica que, en la población femenina, la condición de apego influye en su práctica de salud, por lo que, al estar en una relación, las mujeres tenderían a reducir su actividad física.

Además, al comparar distintos niveles de ejercicio, se encontró un mayor porcentaje de sujetos con pareja en el grupo de nivel de actividad bajo. Esto podría verse apoyado por el estudio de Skoyen et al., (2013), donde se concluye que el involucrarse en actividades físicas y mantener una nutrición saludable podría estar influenciado por la pareja. Sin embargo, estas diferencias no se reflejaron en la comparación de las puntuaciones en IPAQ entre la muestra con y sin pareja.

En nuestro estudio, la puntuación en deseo solitario mostró medias significativamente más altas en la muestra sin pareja. Esto puede relacionarse con lo mencionado por Ortega et al. (2006), donde se explica que el deseo sexual solitario mejora las necesidades sexuales del individuo, mientras que el deseo sexual diádico asumiría un carácter emocional, es decir que velaría por los intereses sexuales de la pareja.

Por otro lado, se observó en la muestra total mayoritariamente un nivel de deseo diádico, lo cual se ha asociado con un buen funcionamiento sexual (Moyano y Sierra, 2014). Con respecto al deseo sexual en las mujeres, este estudio obtuvo cifras significativamente inferiores a las de los hombres. Diversos estudios realizados en mujeres (Finley, 2017; Mehmet et al., 2010; Moyano y Sierra, 2014; Pujols et al., 2010) atribuyen este hecho a factores estresantes como dificultades financieras, presiones académicas, profesionales o la distorsión en la imagen corporal. Por otro lado, se observaron medias significativamente mayores de excitabilidad en los hombres, mientras que en las mujeres se evidenció un aumento en SIS2. Coincidiendo con estos resultados, la investigación realizada por Moyano y Sierra (2014) concluye, que existe mayor puntuación en SES por parte de los hombres debido a la manifestación de fantasías sexuales, conductas sexuales con sus parejas o alta frecuencia en la masturbación. Sin embargo, a diferencia del presente trabajo, las mujeres en el estudio de Moyano y Sierra (2014), obtuvieron un alto puntaje en SIS1, debido a la distracción durante la actividad sexual, mientras que la puntuación es SIS2 referente al riesgo que tiene el sujeto de ser descubierto o al contagio de una infección, resultó similar en ambos sexos. No obstante, la investigación sobre la validez del autoinforme enfatiza sobre la importancia de la deseabilidad social y las normas sociales percibidas que podrían derivar en un sesgo de respuesta (Catania, Gibson, Chitwood, y Coates, 1990). En este sentido, del mismo modo que en los estudios de Fisher (Fisher, 2007; Fisher, 2009) se señalaba a que cierta información en varones estaba influenciada por las normas 
sociales, el presente estudio considera la posibilidad de una mayor "deseabilidad social" en el grupo de hombres, al intentar dar una imagen distorsionada más positiva.

Siguiendo con la comparación de los distintos niveles de ejercicio, los datos muestran que la inhibición sexual relacionada con el miedo a ser descubierto o al contagio de enfermedades (SIS2), obtuvo puntuaciones menores en el grupo con un nivel de actividad alto. A su vez, aquellos sujetos con un nivel de actividad moderado o alto, alcanzan mayores puntuaciones en las escalas de deseo y excitación sexual. Esto concuerda con lo postulado por Huang et al. (2007), Penhollow y Young (2008), y Hackney et al. (2017), quienes mencionan que el ejercicio fomenta la deseabilidad sexual. Sin embargo, el estudio realizado por Sacomori et al. (2013) sobre una muestra femenina no encontró relación significativa entre el deseo y el nivel de ejercicio.

Como limitaciones de este estudio podemos mencionar por un lado la recolección de datos mediante cuestionarios autoadministrados online, generando así un sesgo de deseabilidad y la imposibilidad de los investigadores de reconocer si un mismo sujeto contesta la encuesta en más de una ocasión, así como observar determinadas conductas como el porcentaje de intentos vs porcentaje de encuestas cumplimentadas. Además, este estudio no tuvo en cuenta el tipo de actividad física realizada así como el tipo de deporte (competitivo o no) o el nivel de competición (federado, alto rendimiento o profesional). Del mismo modo, la no inclusión de variables relevantes para los resultados como es la ocupación, el estado civil, el número de hijos o la dedicación horaria a la actividad física-deportiva y actividades extradeportivas pudo suponer también una limitación para este estudio. Así mismo, la clasificación en deseo bajo o normal, o la inclusión de puntuaciones elevadas dentro del grupo con deseo normal, pudo suponer otra limitación en el estudio. Por último, la orientación sexual, que no fue tomada en cuenta en esta investigación, podría de algún modo influir en la relación entre ejercicio y el deseo.

Futuras líneas de investigación podrían considerar el estudio de todas las variables mencionadas anteriormente, así como el análisis de las diferencias con respecto a un grupo con puntuaciones elevadas en deseo sexual o que pudiera presentar cierto nivel de adicción al sexo. Así mismo, resultaría interesante determinar si la relación entre ejercicio y deseo observada en este estudio, se produce de modo similar en otro tipo de poblaciones.

\section{CONCLUSIÓN}

Los resultados del presente estudio sugieren que el deseo sexual está relacionado con el nivel de actividad física, lo que a su vez impacta en la inhibición y excitación sexual del joven adulto. Estos datos nos llevarían a determinar que niveles de actividad media y alta, contribuyen de forma positiva en la conducta sexual de las personas, suponiendo un posible punto de partida para futuras investigaciones capaces de proponer un programa de ejercicio como intervención para aquellas 
personas con problemas del deseo, considerando la importancia de niveles de actividad física adecuados, y su repercusión en la sexualidad. Así mismo, como posibles implicaciones prácticas de los resultados obtenidos en este estudio, cabría señalar el desarrollo de programas de ejercicio moderado dirigido al aumento la libido en grupos de población con bajo deseo sexual.

\section{REFERENCIAS BIBLIOGRÁFICAS}

Adeniyi, AF., Adeleye, JO., y Adeniyi, CY. (2010). Diabetes, Sexual Dysfunction and Therapeutic Exercise: A 20 Year Review. Current Diabetes Reviews, 6(4), 201-206. http://dx.doi.org/10.2174/157339910791658907

Carpenter, D. L., Janssen, E., Graham, C. A., Vorst, H., y Wicherts, J. (2011) The Sexual Inhibition/Sexual Excitation Scales-Short Form (SIS/SES-SF). Handbook of Sexuality-Related Measures, Vol. 3. Publisher: Routledge. pp. 236-239.

Bancroft, J., Graham, C. A., Janssen, E., \& Sanders, S. A. (2009). The dual control model: Current status and future directions. Journal of Sex Research, 46(23), 121-142. http://dx.doi.org/10.1080/00224490902747222

Catania, J. A., Gibson, D. R., Chitwood, D. D., \& Coates, T. J. (1990). Methodological problems in AIDS behavioral research: influences on measurement error and participation bias in studies of sexual behavior. Psychological bulletin, 108(3), 339.

Crespo-Salgado, J., Delgado-Martín, J., Blanco-Iglesias, O., y Aldecoa-Landesa, S. (2015). Guía Básica de detención de sedentarismo y recomendaciones de actividad física en atención primaria. Atención Primaria, 47(3), 175-183. http://dx.doi.org/10.1016/j.aprim.2014.09.004

Corona, G., Rastrelli, G., Jannini, E., Vignozzi, L., Manucci, E., Forti, G., y Maggi, M. (2013). Risk factors associated with primary and secondary reduced libido in male patients with sexual dysfuntion. Endocrine Abstracts. http://dx.doi.org/10.1530/endoabs.32.p672

Corona, G., Isidori, A., Aversa, A., Burnett, A., y Maggi, M. (2016). Endocrinologic Control of Men's Sexual Desire and Arousal/Erection. The Journal Of Sexual Medicine, 13(3), 317-337. http://dx.doi.org/10.1016/j.jsxm.2016.01.007

Davis, S., Sandherg, J., Bradford, A. y Larson, J. (2016). Gender differences in couple attachment behavior as predictors of dietary habits and physical activity levels. Journal Of Health Psychology, 21(12), 3048-3059. http://dx.doi.org/10.1177/1359105315592049

Dosch, A., Ghisletta, P., \& Van der Linden, M. (2016). Body image in dyadic and solitary sexual desire: The role of encoding style and distracting thoughts. The Journal of Sex Research, 53(9), 1193-1206. http://dx.doi.org/10.1080/00224499.2015.1096321

Esposito, K., Ciotola, M., Giugliano, F., Maiorino, M., Autorino, R., De Sio, Giugliano, G., Nicoletti, D’Andrea, y Giugliano, D. (2009). Effects of Intensive Lifestyle Changes on Erectile Dysfunction in Men. The Journal Of Sexual Medicine, 6(1), 243-250. https://doi.org/10.1111/j.1743-6109.2008.01030.x 
Rev.int.med.cienc.act.fís.deporte - vol. 20 - número 77 - ISSN: 1577-0354

European Opinion Research Group. (2003). Physical activity in 15 European states No. 58.2. Brussels: Special Eurobarometer. European Commission.

Finley, N. (2017). Lifestyle Choise Can Augment Female Sexual Well-Being. American Journal Of Lifestyle Medicine, 12(1), 38-41. http://dx.doi.org/10.1177/1559827617740823

Fisher, T. D. (2007). Sex of experimenter and social norms effects on reports of sexual behavior in young men and women. Archives of Sexual Behavior, 26, 89-100. http://dx.doi.org/10.1007/s10508-006-9094-7

Fisher, T.D. (2009). The impact of socially conveyed norms on the reporting of sexual behavior and attitudes by men and women. Journal of Experiemental Social Psychology, 46, 567- 572. http://dx.doi.org/10.1016/j.jesp.2009.02.007

Granados, M.R.; Salinas, J.M.; Sierra, J.C. (2017). Spanish versión of the Sexual Excitation/Sexual Inhibition Inventory For Women: Factorial structure, reliability and validity evidences. International Journal of Clinical and Health Psychology, 17(1), 46-55. http://dx.doi.org/10.1016/j.ijchp.2016.09.003

Haavio-Mannila, E., y Purhonen, S. (2001). Slimness and self-rated sexual attractiveness: Comparisons of men and women in two cultures. Journal Of Sex Research, 38(2), 102-110. http://dx.doi.org/10.1080/00224490109552077

Habel, M., Dittus, P., De Rosa, C., Chung, E., y Kerndt, P. (2010). Daily Participation in Sport and Students'Sexual Activity. Perspectives On Sexual And Reproductive Health, 42(4), 244-250. https://doi.org/10.1363/4224410

Hackney, A., Lane, A., Register-Mihalik, J., y O'Leary, C. (2017). Endurance Exercise Training and Male Sexual Libido. Medicine \& Science in Sport \& Excercise, $\quad 49(7)$, 1383-1388. http://dx.doi.org/10.1249/mss.0000000000001235

Hsiao, W., Shrewsberry, A., Moses, K., Johnson, T., Cai, A., Stuhldreher, P. Dusseault, y Ritenour. (2012). Exercise is Associated with Better Erectile Function in Men Under 40 as Evaluated by the International Index of Erectil Function. The Journal of Sexual Medicine, 9(2), 524-530. http://dx.doi.org/10.1111/j.1743-6109.2011.02560.x

Huang, C., Lee, L., y Chang, M. (2007). The Influences of Personality and Motivation on Exercise Participation and Quality of Life. Social Behavior and Personality: An International Journal, 35(9), 1189-1210. http://dx.doi.org/10.2224/sbp.2007.35.9.1189

Jiménez-Torres, M. G., Godoy-Izquierdo, D. \& Godoy, G. J. F. (2012). Relación entre los motivos para la práctica físico-deportiva y las experiencias de flujo en jóvenes: diferencias en función del sexo. Universitas Psychologica, 11(3), 909-920.

Jiménez, J., Zagalaz, M., Molero, D., Pulido, M., y Ruiz, J. (2013). Capacidad aeróbica, felicidad y satisfacción con la vida en adolescentes españoles. Revista de Psicología del Deporte, 22(2), 429-436. ISSN 1132-239X

Khoo, J., Tian. H., Tan, B., Chew, K., Ng, C., Leong, D., Teo y Chen. (2013). Comparing Effects of Low-and High- Volume Moderate-Intensity Exercise on Sexual Function and Testosterone in Obese Men. The Journal of Sexual Medicine, 10(7), 1823-1832. http://dx.doi.org/10.1111/jsm.12154 
Koyuncu, M., Tok, S., Canpolat, A., Catikkas, F. (2010). Body image satisfaction and dissatisfaction, social physique anxiety, selft-esteem, and body fat ratio in female exercisers and nonexercisers. Social Behavior and Personality: An International Journal, 38(4), 561-570. http://dx.doi.org/10.2224/sbp.2010.38.4.561

Kulig, K., Brener, N., y McManus, T. (2003). Sexual Activity and Substance Use Among Adolescents by Category of Physical Activity Plus Team Sport Participation. Archives of Pediatrics \& Adolescent Medicine, 157(9), 905. http://dx.doi.org/10.1001/archpedi.157.9.905

Lane, A., Register-Mihalik, J., O'Leary, C., y Hackney, A. (2016). Relationship Between Exercise Training and Libido in Endurance Trained Men. Medicine \& Science in Sport \& Exercise, 48, 267. http://dx.doi.org/ 10.1249/01.mss.0000485803.44765.41

La Vignera, S., Condorelli, R., Vicari, E., D’Agata, R., y Calogero, A. (2011). Aerobic physical activity improves endotelial function in the middle-aged patients with erectile dysfunction. The Aging Male, 14(4), 265-272. http://dx.doi.org/10.3109/13685538.2010.544344

La Vignera, S., Condorelli, R., Vicari, E., D’Agata, R., y Calogero, A. (2012). Physical Activity and Erectile Dysfunction in Middle-Aged Men. Journal Of Andrology, 33(2), 154-161. http://dx.doi.org/10.2164/jandrol.111.013649

Lorenz, T., Meston, C. (2012). Acute Exercise Improves Physical Sexual Arousal in Women Taking Antidepressants. Annals of Behavioral Medicine, 43(3), 352-361. http://dx.doi.org/10.1007/s12160-011-9338-1

Lorenz, T., Meston, C. (2013). Exercise Improves Sexual Function in Women Taking Antidepressants: Results From a Randomized Crossover Trial. Depression and Anxiety, 31(3), 188-195. http://dx.doi.org/10.1002/da.22208

Maio, G., Saraeb, S., y Marchiori, A. (2010). Physical Activity and PDE5 Inhibitors in the Treatment of Erectile Dysfunction: Results of a Randomized Controlled Study. The Journal of Sexual Medicine, 7(6), 2201-2208. https://doi.org/10.1111/j.1743-6109.2010.01783.x

Miller, K., Sabo, D., Farrell, M., Barnes, G., y Melnick, M. (1998). Athletic Participation and Sexual Behavior in Adolescents: The Different Worlds of Boys and Girls. Journal of Health and Social Behavior, 39(2), 108. http://dx.doi.org/10.2307/2676394

Mor, Z. Parfionov, K., Davidovitch, N., Grotto, I. (2014). Gym exercising patterns, lifestyle and high-risk sexual behaviour in men who have sex with men and in heterosexual men. BMJ Open, 4(11), e005205. http://dx.doi.org/10.1136/bmjopen-2014-005205

Moyano, N., y Sierra, J. (2014). Validación de las Escalas de Inhibición Sexual/Excitación Sexual- Forma Breve (SIS/SES-SF). Terapia Psicológica, 32(2), 87-100. http://dx.doi.org/10.4067/S0718-48082014000200002

Nogueira Avelar e Silva, R., Wijtzes, A., van deBongardt, D., van de Looij-Jansen, P., Bannink, R., y Raat, H. (2016). Early Sexual Intercourse: Prospective Associations with Adolescents Physical Activity and Screen Time. PLOS ONE, 11(8), e0158648. http://dx.doi.org/10.1371/journal.pone.0158648 
Ortega, V., Zubeidat, I., y Sierra, J. (2006). Further Examination of Measurement Properties of Spanish Version of the Sexual Desire Inventory with Undergraduates and Adolescent Students. Psycological Reports, 99(1), 147165. http://dx.doi.org/10.2466/pr0.99.1.147-165

Penhollow, T., y Young, M. (2008) Predictors of Sexual Satisfaction: The Role of Body Image and Fitness. Electronic Journal of Human Sexuality, 11.

Pujols, Y., Meston, C., y Seal, B. (2010). The Association Between Sexual Satisfaction and Body Image in Women. The Journal of Sexual Medicine, 7(2), 905-916. http://dx.doi.org/10.1111/j.1743-6109.2009.01604.x

Román-Viñas, B., Ribas-Barba, L., Ngo, J., \& Serra-Majem, L. (2013). Validación en población catalana del cuestionario internacional de actividad física. Gaceta Sanitaria, 27(3), 254-257. https://doi.org/10.1016/j.gaceta.2012.05.013

Sacomori, C.; Felizola, F.L.V.; Kruguer, A.P.; Sperandio, F.F. y Cardoso, F.L. (2013) Nivel de actividad física y función sexual de mujeres / Physical activity level and sexual function of women. Revista Internacional de Medicina y Ciencias de la Actividad Física y el Deporte, 13(52), 703-717. http://cdeporte.rediris.es/revista/revista52/artinfluencia420.htm

Sexual Desire Quiz. Maze Women's Sexual Health. (2018). Recuperado de https://www.mazewomenshealth.com/whats-new/resources/sexual-desireinventory/

Sigmundová, D., Sigmund, E., Hamrík, Z., Kalman, M., Pavelka, J., \& Frömel, K. (2015). Sedentary behaviour and physical activity of randomized sample of czech adults aged 20-64 years: ipaq and gpaq studies between 2002 and 2011. Central European Journal of Public Health, 23,S91-S96 http://dx.doi.org/10.21101/cejph.a4142

Skoyen, J. Blank, E., Corkery, S. y Butler, E. (2013). The interplay of partner and individual values predicts daily fluctuations in eating and physical activity. Journal Of Social and Personal Relationships 30(8), 1000-1019. http://dx.doi.org/10.1177/0265407513479214

Sgrò, P., y Di Luigi, L. (2017). Sport and Male Sexuality. Jounal Of Endocrinological Investigation, 40(9), 911-923. http://dx.doi.org/10.1007/s40618-017-0652-8

Spector, I., Carey, M., y Steinberg, L. (1996). The sexual desire inventory: Development, factor structure, and evidence of reliability. Journal Of Sex \& Marital Therapy, 22(3), 175-190. http://dx.doi.org/10.1080/00926239608414655

Stojanovska, L., Apostolopouslos, V., Polman, R., y Borkoles, E. (2014). To exercise, or, not to exercise, during menopause and beyond. Maturitas, 77(4), 318-323. http://dx.doi.org/10.1016/j.maturitas.2014.01.006

Taliaferro, L., Rienzo, B., y Donovan, K. (2010). Relationships Between Youth Sport Participation and Selected Health Risk Behaviors From 1999 to 2007. Journal Of School Health, 80(8), 399-410. https://doi.org/10.1111/j.17461561.2010.00520.x

UhImann, L. R., Donovan, C. L., Zimmer-Gembeck, M. J., Bell, H. S., y Ramme, R. A. (2018). The fit beauty ideal: A healthy alternative to thinness or a wolf in 
Rev.int.med.cienc.act.fís.deporte - vol. 20 - número 77 - ISSN: 1577-0354

sheep's clothing?. Body image, 25, 23-30. http://dx.doi.org/ 10.1016/j.bodyim.2018.01.005

Número de citas totales / Total references: 48 (100\%)

Número de citas propias de la revista / Journal's own references: $1(2,1 \%)$

Rev.int.med.cienc.act.fís.deporte - vol. 20 - número 77 - ISSN: 1577-0354 


\section{ANEXO}

Cuestionario socio-demográfico

Edad (años)

¿Padece alguna limitación física que impida o afectaran de algún modo a la realización de actividad física?

1] Sí

No

¿Padece alguna enfermedad crónica que impida o afectaran de algún modo a la realización de actividad física?

$\square$ Sí

$\square$ No

Género

Hombre

Mujer

¿Tienes pareja estable actualmente? (más de tres meses)

Sí

No

Mantengo una relación íntima que no considero pareja

Nivel de estudios

Elemental (ESO, Certificado de Estudios Primarios, ...)

Medio (Bachillerato, Ciclos formativos medio y superior,....)

Superiores (Universitarios: grado, máster, doctorado...)

Sin estudios

Rev.int.med.cienc.act.fís.deporte - vol. 20 - número 77 - ISSN: 1577-0354 